\title{
Association between carotenoids and prostate cancer risk: A Meta-Analysis
}

\author{
Hadjer BENKHADDA ${ }^{1}$, Manel BOUCHENTOUF ${ }^{1}$, Salah Eddine EL HERRAG ${ }^{1}$ * \\ ${ }^{1}$ Department of Biology, Faculty of Natural and Life Sciences, Djillali Liabes University, PO Box 89, Sidi-Bel-Abbes (22000), Algeria
}

\section{A R T I C LE INFO}

\section{Article history:}

Received 11 November 2018

Accepted 20 January 2019

Available online 17 February 2019

\section{Keywords:}

Prostate cancer

Carotenoids

Lycopene, $\beta$-Carotene

$\alpha$-Carotene, $\beta$-cryptoxanthin

Lutein/zeaxanthin,

Meta-analysis.

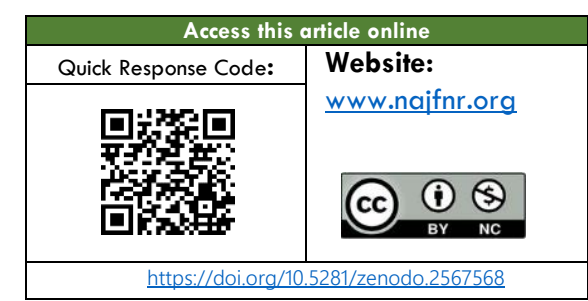

\author{
A B S T R A C T
}

Background: Carotenoids have been hypothesized to promote the prevention and treatment of prostate cancer (PC). Aims: Conducting a meta-analysis to assess whether the association differs by the type of carotenoids and to compare the relationships of each type of carotenoid with PC risk. Methods and Material: A literature search was conducted using the PubMed database. Pertinent studies were included in the metaanalysis of higher versus lower categories of dietary intakes or serum concentrations of carotenoids in relation to PC. A random-effects model was used to obtain the pooled odds ratios (ORs) and their confidence intervals (95\%) of the case-control and cohort studies, for generic inverse variance data. Results: 13 identified studies involved 44,861 participants. The meta-analysis determined, for total carotenoids and lycopene, a statistically significant relationship with PC risk; OR: $0.88 ; 95 \% \mathrm{Cl} 0.81-0.96$ and $0.87 ; 95 \%$ $\mathrm{Cl}$ 0.77-0.99 respectively. However, no other carotenoid was noticed to have a statistically significant association with $\mathrm{PC}$ risk. Comparison of the effect of each type of carotenoid on PC risk showed that lycopene, lutein-zeaxanthin, and $\beta$-cryptoxanthin had a moderate inverse but not statistically significant relationship with PC risk. Compared to $\beta$-carotene, and $\alpha$-carotene results, their effect estimates were higher than the previous carotenoids. Conclusions: Our findings indicate that PC was inversely associated with total carotenoids and lycopene dietary intake and serum concentrations. These data provide support for the utilization of carotenoids for the treatment and prevention of PC.

Article edited by Pr. Meghit Boumediene KHALED and Dr. Mickaël RIALLAND

*Corresponding author Tel:+213697813902_salaheddine.elherrag@univ-sba.dz

\section{INTRODUCTION}

Prostate cancer (PC) or carcinoma of the prostate is ranked as the second most common cancer within men worldwide [1]. An estimated number of 1.3 million cases were diagnosed worldwide with PC in 2018, accounting for $13.5 \%$ of the cancers diagnosed in men, with almost $63 \%$ of them (802.294) occurring in more developed regions [2]. The development of PC is complex and influenced by a combination of genetic, hormonal, and social factors. Carotenoids represent biologically active phytochemicals which are present as micro-components in fruits and vegetables and are responsible for their yellow, orange and red colors [3]. These micronutrients are thought to be responsible for the beneficial properties of fruits and vegetables in preventing human diseases including cardiovascular diseases [4], cancer [5-7], and other chronic diseases, and have distinct anti-oxidative properties, including protecting DNA and other important biomolecules from free radicals [8]. In recent years the antioxidant properties of carotenoids have been the major focus of research. More than 600 carotenoids have so far 
been identified in nature. However, only about 40 are present in a typical human diet. Among these 40, about 20 carotenoids have been identified in human blood and tissues. Close to $90 \%$ of the carotenoids, in the diet and the human body, is represented by alpha-carotene, betacarotene, lutein, beta-cryptoxanthin, and lycopene [9]. Higher consumption of tomato products, dietary lycopene intake, and circulating lycopene concentrations were found to be inversely associated with prostate cancer risk $[10,11]$. Many epidemiological studies have investigated the association between prostate cancer and carotenoids, however, results have been inconsistent [12,13]. Consequently, In the current study, we aimed to investigate the relationship between dietary intake or blood concentrations of these carotenoids and their potential preventive effect on PC.

\section{MATERIAL AND METHODS}

\subsection{Search strategy}

The current meta-analysis was performed in accordance with PRISMA guidelines [14]. The literature search was independently undertaken by two authors (H.B and M.B). The author (S.E) made the final decision in case of any discrepancy. A comprehensive literature search was conducted using the PubMed database [https://www.ncbi.nlm.nih.gov/pubmed] to identify relevant English published articles on the relationship between carotenoids dietary intake or supplementation and the risk of PC. Using medical subject heading (Mesh), with the key terms: "carotenoids" and "prostatic neoplasms". English full texts were searched with no population restriction (the final search was performed on 13 May 2018).

\subsection{Study selection}

Studies that met the following criteria were included in the meta-analysis: (a) the study design was a cohort, and casecontrol; (b) the primary outcome was clearly defined as prostate cancer; (c) studies that assessed the relationship between carotenoids (lycopene, $\beta$-carotene, $\alpha$-carotene, $\beta$ cryptoxanthin, and lutein/zeaxanthin) and PC risk ; (d) the exposure of interest was carotenoids intake and supplementation; (e) the study provided Odds Ratios (OR) with 95\% confidence intervals $(\mathrm{Cl})$ for exposure categories. Furthermore, letters, comments, and articles, not published as full text, reviews, meta-analyses, animal studies, cellular culture, reports, and articles with missing or not appropriate data, were excluded.

\subsection{Data extraction}

Data collection form, for each publication, was as follow: first author's name, year of publication, country of origin, outcomes, recruitment and intervention periods, study type, number of cases, controls, total number, and age of participants in the study, exposure values of carotenoids (serum and/or intake), and the adjusted confounders.

\subsection{Summary measures}

Review Manager 5.3 was utilized to analyze the data [15]. The pooled OR with $95 \% \mathrm{Cl}$ is given both for the fixed effects model and the random effects model. If value 1 is not within the $95 \% \mathrm{Cl}$, then the OR is statistically significant at the $5 \%$ level $(P \leq 0.05)[16]$. We used the study-specific OR for the highest versus lowest categories of dietary carotenoids intake or carotenoids concentration exposure for the meta-analysis. The study selection, data extraction, and statistical analysis were independently undertaken by two authors (H.B and M.B), a third author (S.E) made the final decision in case of any disagreement.

\subsection{Publication bias}

Publication bias and the small study effect were assessed using Begg's [17] rank correlation test and Egger's regression test [18], following a visual assessment of funnel plots. The $\mathrm{R}$ program was used for assessing the bias risk across studies [19].

\subsection{Sensitivity and subgroup analyses}

Pre-Specified subgroup analyses were performed allowing for study types, location, methods used, and the adjusted confounders. Furthermore, sensitivity analyses were performed by excluding one dataset at a time with the purpose of analyzing the influence of each study on the effect estimates and the heterogeneity.

\section{RESULTS}

\subsection{Study selection}

The literature search yielded a total of 754 publications. After screening, 13 studies were included and 741 were excluded, among the 741 excluded articles, 470 did not meet the criteria, 194 reviews, and meta-analysis, and 34 letters to editorial and comments, and 43 not appropriate or missing data (Figure 1).

\subsection{Study characteristics}

Table 1 summarizes properties of the included studies. Two cohort and 11 case-control studies were included. Seven studies were carried out in the USA [12, 13, 20-24], three in Australia [25-27], two in Europe [28, 29], and one in Asia[30]. Among the included studies, eleven studies reported the effects of dietary intake or serum concentrations of lycopene and $\beta$-Carotene on PC. With the exception of five studies, almost all adjusted analyses 
included age as a potential confounder and four studies controlled for family history of PC and body mass index (BMI).

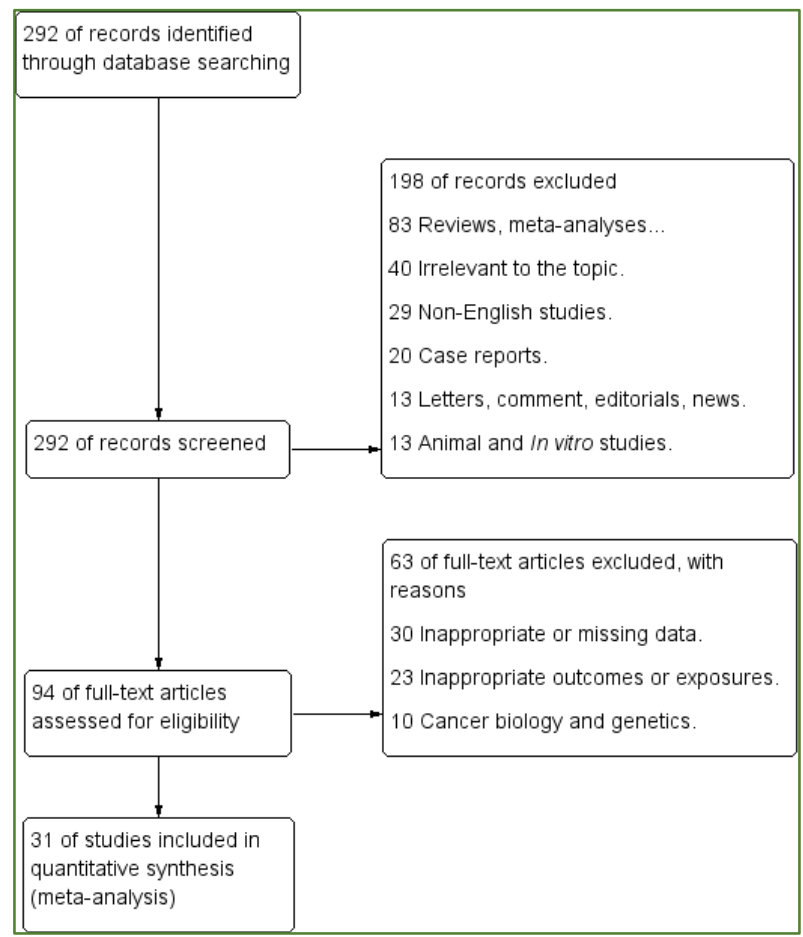

Figure 1: Flowchart of study selection

\subsection{Relationship between carotenoids and risk of PC}

The results of the meta-analysis, of highest versus lowest categories of carotenoids intake and serum concentrations, are summarized in table 2 and figure 2. A total of 44.861 participants from these studies were assigned to intervention or control groups. The number of participants in each case-control and cohort ranged from 321 to 29.361. The effects of lycopene and $\beta$-carotene were examined singly or in combination in six studies and in combination with other carotenoids in seven studies.

The summary effect of the main analysis on PC was statistically significant with an OR of 0.88 (95\% Cl 0.81-0.96), with moderate heterogeneity $\left(I^{2}=56 \%\right)$. Thus, we conclude that carotenoids intake decreased the overall relative risk of PC.

Nonetheless, we observed, after performing a sensitivity analysis, that one study (Jian et al., 2004) [30] constituted the major source of heterogeneity in our meta-analysis and after neglecting this study, $\mathrm{I}^{2}$ dropped to only $2 \%$.

\subsubsection{Lycopene}

A total of 11 studies with 15 datasets, on the relationship between lycopene and prostate cancer, were included. Our results showed that lycopene significantly reduced the prostate cancer risk $(\mathrm{OR}=0.88 ; 95 \% \mathrm{Cl} 0.78-1.00)$, with moderate heterogeneity $\left(I^{2}=44 \%\right)$.
According to further subgroup analysis, as shown in Table 3, we did not identify significant associations between PC risk and lycopene for cohort studies $(P=0.56)$. However, case-control studies indicated that lycopene significantly decreased $\mathrm{PC}$ risk (OR $=0.87 ; 95 \% \mathrm{Cl}$ 0.77-0.99). Moreover, studies conducted in Australia and North American countries showed a stronger inverse relationship than European studies (OR $=0.76 ; 95 \% \mathrm{Cl} 0.53-1.09)$, $(\mathrm{OR}=0.90$; $95 \% \mathrm{Cl} 0.83-0.99, \mathrm{P}$ (Z-test) $=0.03)$, and $(\mathrm{OR}=0.94 ; 95 \%$ $\mathrm{Cl} 0.75-1.16)$ respectively. Five studies with seven datasets reported data about dietary lycopene supplementation and $\mathrm{PC}$ risk. A statistically significant negative association was observed $(\mathrm{OR}=0.83 ; 95 \% \mathrm{Cl} 0.70-0.99)$. A less strong and yet statistically non-significant inverse relationship was found between serum lycopene concentrations and PC (OR $=0.96 ; 95 \% \mathrm{Cl} 0.81-1.15)$.

\subsection{2 $\alpha$-Carotene}

Regarding the overall pooled estimates of the association between $\alpha$-carotene and PC risk, we noticed a statistically non-significant negative association with an OR of 0.80 (95\% Cl 0.60-1.07) and a moderate heterogeneity $\left(I^{2}=59\right.$ $\%)$. Alpha carotene was found to decrease PC risk in casecontrol studies and in studies conducted in North American countries $(\mathrm{OR}=0.86 ; 95 \% \mathrm{Cl} 0.64-1.15)$ and $(\mathrm{OR}=0.87$; $95 \% \mathrm{Cl} 0.59-1.29)$ respectively, although the associations were not statistically significant. The same pattern was noticed for studies investigating the effect of highest versus lowest categories of $\alpha$-carotene intake (OR $=0.87 ; 95 \% \mathrm{Cl}$ $0.59-1.29)$ and serum concentrations $(O R=0.65 ; 95 \% \mathrm{Cl}$ $0.33-1.27)$.

\subsection{3 $\beta$-Carotene}

A total of 11 studies, including 15 datasets, reported data on $\beta$-carotene and $P C$ risk. Comparing the highest with the lowest dietary intake or blood concentrations, a statistically non-significant inverse association was found with an OR of 0.95 (95\% Cl 0.78-1.11) and moderate heterogeneity $\left(\mathrm{I}^{2}=54 \%\right)$. The main analysis was stratified according to study type, population, and methods used to assess the relationship. Therefore, nine studies with 13 datasets of case-control studies were pooled $(\mathrm{OR}=0.95 ; 95 \% \mathrm{Cl} 0.78$ 1.16), and two cohort studies ( $O R=0.79 ; 95 \% \mathrm{Cl} 0.46-1.35)$, suggesting a statistically non-significant negative association between $\beta$-carotene and PC risk. Our findings suggest that $\beta$-carotene has no effect on $P C$ risk among North American (OR $=1.02 ; 95 \% \mathrm{Cl} 0.83-1.26)$, and Australian populations $(\mathrm{OR}=1.00 ; 95 \% \mathrm{Cl} 0.76-1.31)$ and studies examining the effect of highest versus lowest categories of serum concentrations of $\beta$-carotene on PC $(\mathrm{OR}=1.01 ; 95 \% \mathrm{Cl} 0.84-1.23)$. 


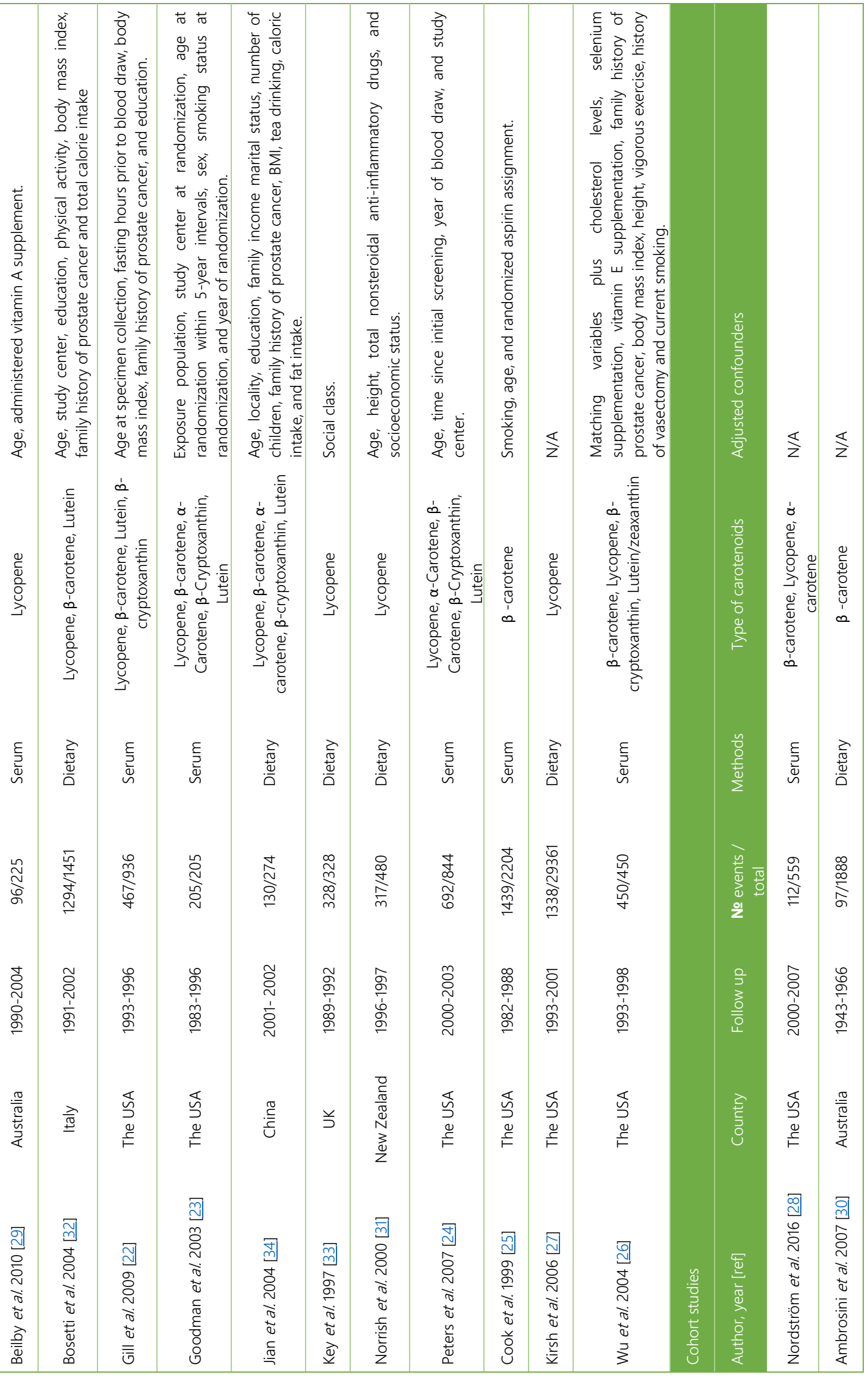


Table 2: Summary of results

\begin{tabular}{|c|c|c|c|c|c|c|c|c|c|}
\hline \multirow{2}{*}{ Analysis } & \multirow{2}{*}{ № of studies (datasets) [ref] } & \multirow{2}{*}{ Model } & \multirow{2}{*}{ OR $[95 \% \mathrm{Cl}]$} & \multirow{2}{*}{ Z-test } & \multicolumn{3}{|c|}{ Heterogeneity } & \multicolumn{2}{|c|}{$\begin{array}{c}\text { Publication bias } \\
\text { (P value) }\end{array}$} \\
\hline & & & & & $I^{2}(\%)$ & Tau 2 & $\mathrm{Chi}^{2}$ & $\begin{array}{c}\text { Begg's } \\
\text { test }\end{array}$ & $\begin{array}{c}\text { Egger's } \\
\text { test }\end{array}$ \\
\hline Lycopene & 11 (15) $[22-24,26-29,31-\underline{34}]$ & RE & $0.88[0.78-1.00]$ & $\begin{array}{c}1.97 \\
(P=0.05)\end{array}$ & 70 & 0.12 & $\begin{array}{l}19.74, \mathrm{df}=6 \\
(P=0.003)\end{array}$ & 0.9605 & 0.678 \\
\hline$\beta$-Carotene & 11 (15) $[\underline{22}-\underline{26,28}-\underline{32,34]}$ & RE & $0.93[0.78-1.11]$ & $\begin{array}{c}0.78 \\
(P=0.43)\end{array}$ & 54 & 0.06 & $\begin{array}{c}30.12, \mathrm{df}=14 \\
(P=0.007)\end{array}$ & 0.216 & 0.522 \\
\hline Lutein/Zeaxanthin & $6(9)[\underline{22}-\underline{24,26,32,34]}$ & RE & $0.86[0.64-1.15]$ & $\begin{array}{c}1.01 \\
(P=0.31)\end{array}$ & 72 & 0.13 & $\begin{array}{l}28.26, \mathrm{df}=8 \\
(P=0.0004)\end{array}$ & \multicolumn{2}{|c|}{ NA } \\
\hline$\beta$-cryptoxanthin & $6(7)[\underline{22}-\underline{24,26,32,34}]$ & RE & $0.80[0.58-1.09]$ & $\begin{array}{c}1.42 \\
(P=0.16)\end{array}$ & 70 & 0.12 & $\begin{array}{l}19.74, \mathrm{df}=6 \\
(P=0.003)\end{array}$ & \multicolumn{2}{|c|}{ NA } \\
\hline$\alpha$-Carotene & $6(6)[\underline{23}, 24,26,28,32,34]$ & RE & $0.80[0.60-1.07]$ & $\begin{array}{c}1.47 \\
(P=0.14)\end{array}$ & 59 & 0.07 & $\begin{array}{l}12.16, \mathrm{df}=5 \\
(P=0.03)\end{array}$ & \multicolumn{2}{|c|}{ NA } \\
\hline
\end{tabular}

NA not applicable, $O R$ odds ratio, RE random effects.

Table 3: Subgroup analysis results of the association between Lycopene and prostate cancer

\begin{tabular}{|c|c|c|c|c|c|c|c|c|}
\hline \multirow[b]{2}{*}{ Subgroups } & \multirow{2}{*}{$\begin{array}{l}\text { № of } \\
\text { studies } \\
\text { (№ of } \\
\text { datasets) }\end{array}$} & \multirow[b]{2}{*}{ References } & \multirow[b]{2}{*}{ Model } & \multirow[b]{2}{*}{ OR $[95 \% \mathrm{Cl}]$} & \multirow[b]{2}{*}{ Z- test } & \multicolumn{3}{|c|}{ Heterogeneity } \\
\hline & & & & & & $\begin{array}{c}I^{2} \\
(\%)\end{array}$ & $T^{2}$ & $\mathrm{X}^{2}$ \\
\hline All studies & $11(15)$ & {$\left[\underline{22-} \frac{24,26}{31-34]}\right.$} & $\mathrm{RE}$ & 0.88 [0.78-1.00] & $\begin{array}{c}1.97 \\
(P=0.05)\end{array}$ & 44 & 0.02 & $\begin{array}{c}24.93, \mathrm{df}=14 \\
(P=0.04)\end{array}$ \\
\hline \multicolumn{9}{|l|}{ Study type } \\
\hline Cohort & $1(1)$ & {$[28]$} & $\mathrm{RE}$ & $1.22[0.62-2.40]$ & $\begin{array}{c}0.58 \\
(P=0.56)\end{array}$ & \multicolumn{3}{|c|}{$\mathrm{N} / \mathrm{A}$} \\
\hline Case-control & $12(14)$ & $\frac{[22-24,26,27}{29,31-34]}$ & RE & 0.87 [0.77-0.99] & $\begin{array}{c}2.08 \\
(P=0.04)\end{array}$ & 46 & 0.02 & $\begin{array}{c}24.06, \mathrm{df}=13 \\
(P=0.03)\end{array}$ \\
\hline \multicolumn{9}{|l|}{ Study location } \\
\hline Asia & $1(1)$ & [34] & $\mathrm{RE}$ & $0.18[0.08-0.40]$ & $\begin{array}{c}4.08 \\
(P<0.0001)\end{array}$ & \multicolumn{3}{|c|}{$\mathrm{N} / \mathrm{A}$} \\
\hline Europe & $2(2)$ & {$[32,33]$} & $\mathrm{FE}$ & $0.94[0.75-1.16]$ & $\begin{array}{c}0.59 \\
(P=0.56)\end{array}$ & 0 & $\mathrm{~N} / \mathrm{A}$ & $\begin{array}{c}0.00, \mathrm{df}=1 \\
(P=0.96)\end{array}$ \\
\hline North America & $6(10)$ & $\frac{\left[22-\frac{24,26-}{28]}\right.}{\underline{28}}$ & $\mathrm{FE}$ & 0.90 [0.83-0.99] & $\begin{array}{c}2.22 \\
(P=0.03)\end{array}$ & 5 & $\mathrm{~N} / \mathrm{A}$ & $\begin{array}{c}9.43, \mathrm{df}=9 \\
(P=0.40)\end{array}$ \\
\hline Australia & $2(2)$ & {$[29,31]$} & $\mathrm{FE}$ & $0.76[0.53-1.09]$ & $\begin{array}{c}1.47 \\
(P=0.14)\end{array}$ & 0 & $\mathrm{~N} / \mathrm{A}$ & $\begin{array}{c}0.00, \mathrm{df}=1 \\
(P=0.98)\end{array}$ \\
\hline \multicolumn{9}{|l|}{ Methods } \\
\hline Serum & $6(8)$ & $\frac{[22-24,26,28,}{\underline{29}]}$ & $\mathrm{FE}$ & $0.96[0.81-1.15]$ & $\begin{array}{c}0.41 \\
(P=0.68)\end{array}$ & 0 & $\mathrm{~N} / \mathrm{A}$ & $\begin{array}{c}6.31, \mathrm{df}=7 \\
(P=0.50)\end{array}$ \\
\hline Dietary & $5(7)$ & {$[27,31-34]$} & $\mathrm{RE}$ & $0.83[0.70-0.99]$ & $\begin{array}{c}2.05 \\
(P=0.04)\end{array}$ & 66 & 0.03 & $\begin{array}{c}17.49, \mathrm{df}=6 \\
(P=0.008)\end{array}$ \\
\hline \multicolumn{9}{|l|}{ Adjusted confounders } \\
\hline Age & $8(12)$ & $\frac{[22-24,27,29}{31,32,34]}$ & $\mathrm{RE}$ & 0.88 [0.76-1.01] & $1.77(P=0.08)$ & 52 & 0.03 & $\begin{array}{c}22.85, \mathrm{df}=11 \\
(P=0.02)\end{array}$ \\
\hline $\begin{array}{l}\text { Family history of } \\
\text { PC }\end{array}$ & $4(5)$ & $\frac{[22,26,32}{\underline{34}]}$ & $\mathrm{RE}$ & $0.70[0.44-1.13]$ & $1.45(P=0.15)$ & 76 & 0.20 & $\begin{array}{c}16.96, \mathrm{df}=4 \\
(P=0.005)\end{array}$ \\
\hline BMI & $4(5)$ & $\frac{[22,26,32}{34]}$ & $\mathrm{RE}$ & 0.70 [0.44-1.13] & $\begin{array}{c}1.45 \\
(P=0.15)\end{array}$ & 76 & 0.20 & $\begin{array}{c}16.96, \mathrm{df}=4 \\
(P=0.005)\end{array}$ \\
\hline Education & $3(4)$ & {$[22,32,34]$} & RE & $0.71[0.39-1.30]$ & $\begin{array}{c}1.10 \\
(P=0.27)\end{array}$ & 82 & 0.28 & $\begin{array}{l}16.37, \mathrm{df}=3 \\
(P=0.0010)\end{array}$ \\
\hline Study Center & $3(3)$ & {$[23,24,32]$} & $\mathrm{FE}$ & $1.01[0.85-1.21]$ & $\begin{array}{c}0.16 \\
(P=0.87)\end{array}$ & 0 & $\mathrm{~N} / \mathrm{A}$ & $\begin{array}{c}0.82, \mathrm{df}=2 \\
(P=0.67)\end{array}$ \\
\hline Smoking & $2(2)$ & {$[23,26]$} & $\mathrm{FE}$ & $0.83[0.57,1.21]$ & $\begin{array}{c}0.96 \\
(P=0.34)\end{array}$ & 28 & $\mathrm{~N} / \mathrm{A}$ & $\begin{array}{l}1.38, \mathrm{df}=1 \\
(P=0.24)\end{array}$ \\
\hline Height & $2(2)$ & {$[26,31]$} & FE & $0.72[0.51,1.01]$ & $\begin{array}{c}1.91 \\
(P=0.06)\end{array}$ & 0 & $\mathrm{~N} / \mathrm{A}$ & $\begin{array}{l}0.17, \mathrm{df}=1 \\
(P=0.68)\end{array}$ \\
\hline Caloric intake & $2(2)$ & {$[\underline{32}, 34]$} & RE & $0.43[0.09,2.17]$ & $\begin{array}{c}1.02 \\
(P=0.31)\end{array}$ & 93 & 1.27 & $\begin{array}{l}13.99, \mathrm{df}=1 \\
(P=0.0002)\end{array}$ \\
\hline
\end{tabular}

$B M I$ Body mass index, df degree of freedom, FE fixed effects, N/A not-applicable, $P C$ prostate cancer, $O R$ odds ratio, $R E$ random effects. 


\begin{tabular}{|c|c|c|c|c|c|c|}
\hline \multirow{2}{*}{$\frac{\text { Study or Subgroup }}{\text { 1.1.1 } \alpha \text {-carotene }}$} & log[Odds Ratio] & SE & Weight & \multirow[t]{2}{*}{$\begin{array}{c}\text { Odds Ratio } \\
\text { Iv, Random, } 95 \% \mathrm{CI}\end{array}$} & \multicolumn{2}{|c|}{$\begin{array}{c}\text { Odds Ratio } \\
\text { IV, Random, 95\% Cl }\end{array}$} \\
\hline & & & & & & \\
\hline Bosetti 2004 & -0.1574 & 0.1347 & $3.0 \%$ & $0.85[0.66,1.11]$ & & \\
\hline Goodman 2003 & 0.1655 & 0.2812 & $1.6 \%$ & $1.18[0.68,2.05]$ & & \\
\hline Jian 2004 & -0.8555 & 0.3564 & $1.2 \%$ & $0.43[0.21,0.85]$ & & \\
\hline Nordström 2016 & -0.6633 & 0.3315 & $1.3 \%$ & $0.52[0.27,0.99]$ & & \\
\hline Peters 2007 & 0.1655 & 0.1674 & $2.6 \%$ & $1.18[0.85,1.64]$ & & - \\
\hline $\begin{array}{l}\text { Wu } 2004 \\
\text { Subtotal }(95 \% \mathrm{Cl})\end{array}$ & -0.4075 & 0.2539 & $\begin{array}{l}1.8 \% \\
114 \%\end{array}$ & $0.67[0.40,1.09]$ & & \\
\hline \multicolumn{7}{|c|}{$\begin{array}{l}\text { Heterogeneity: } \operatorname{Tau}^{2}=0.07 ; \mathrm{Chi}^{2}=12.16, \mathrm{df}=5(\mathrm{P}=0.03) ; \mathrm{l}^{2}=59 \% \\
\text { Test for overall effect: } Z=1.47(P=0.14)\end{array}$} \\
\hline \multicolumn{7}{|l|}{ 1.1.2 $\beta$-carotene } \\
\hline Ambrosini 2007 & -0.0101 & 0.2254 & $2.0 \%$ & $0.99[0.64,1.54]$ & 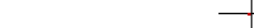 & - \\
\hline Beilby 2010 & -0.181 & 0.315 & $1.4 \%$ & $0.83[0.45,1.55]$ & & \\
\hline Bosetti 2004 & -0.3285 & 0.1374 & $3.0 \%$ & $0.72[0.55,0.94]$ & & \\
\hline Cook 1999 & 0.2869 & 0.1972 & $2.3 \%$ & $1.33[0.91,1.96]$ & & \\
\hline Cook 1999 & 0.2851 & 0.2957 & $1.5 \%$ & $1.33[0.74,2.37]$ & & \\
\hline Cook 1999 & -0.0015 & 0.2906 & $1.5 \%$ & $1.00[0.56,1.76]$ & & \\
\hline Gill 2009 & -0.2107 & 0.1933 & $2.3 \%$ & $0.81[0.55,1.18]$ & & - \\
\hline Gill 2009 & -0.3589 & 0.4067 & $0.9 \%$ & $0.70[0.31,1.55]$ & & \\
\hline Goodman 2003 & -0.1624 & 0.2863 & $1.5 \%$ & $0.85[0.49,1.49]$ & & 一 \\
\hline Jian 2004 & -1.0788 & 0.3611 & $1.1 \%$ & $0.34[0.17,0.69]$ & & \\
\hline Nordström 2016 & -0.5754 & 0.329 & $1.3 \%$ & $0.56[0.30,1.07]$ & & \\
\hline Norrish 2000 & 0.0862 & 0.2084 & $2.2 \%$ & $1.09[0.72,1.64]$ & & \\
\hline Peters 2007 & 0.2624 & 0.1717 & $2.6 \%$ & $1.30[0.93,1.82]$ & & \\
\hline Peters 2007 & 0.5128 & 0.2489 & $1.8 \%$ & $1.67[1.03,2.72]$ & & \\
\hline Wu 2004 & -0.2485 & 0.2426 & $1.9 \%$ & $0.78[0.48,1.25]$ & & - \\
\hline Subtotal $(95 \% \mathrm{Cl})$ & & & $27.4 \%$ & $0.93[0.78,1.11]$ & & \\
\hline \multicolumn{7}{|c|}{$\begin{array}{l}\text { Heterogeneity: } \text { Tau }^{2}=0.06 ; \mathrm{Chi}^{2}=30.12, \mathrm{df}=14(\mathrm{P}=0.007) ; \mathrm{I}^{2}=54 \% \\
\text { Test for overall effect: } Z=0.78(\mathrm{P}=0.43)\end{array}$} \\
\hline \multicolumn{7}{|l|}{ 1.1.3 $\beta$-cryptoxanthin } \\
\hline Bosetti 2004 & -0.1059 & 0.1319 & $3.0 \%$ & $0.90[0.69,1.16]$ & - & \\
\hline Gill 2009 & -0.0305 & 0.198 & $2.3 \%$ & $0.97[0.66,1.43]$ & - & 一 \\
\hline Gill 2009 & -0.1393 & 0.4196 & $0.9 \%$ & $0.87[0.38,1.98]$ & & \\
\hline Goodman 2003 & -0.2107 & 0.29 & $1.5 \%$ & $0.81[0.46,1.43]$ & & - \\
\hline Jian 2004 & -1.9065 & 0.4223 & $0.9 \%$ & $0.15[0.06,0.34]$ & & \\
\hline Peters 2007 & 0.0862 & 0.1697 & $2.6 \%$ & $1.09[0.78,1.52]$ & & - \\
\hline Wu 2004 & -0.0619 & 0.265 & $1.7 \%$ & $0.94[0.56,1.58]$ & & \\
\hline Subtotal $(95 \% \mathrm{Cl})$ & & & $12.9 \%$ & $0.80[0.58,1.09]$ & & \\
\hline \multicolumn{7}{|c|}{$\begin{array}{l}\text { Heterogeneity: } \text { Tau }^{2}=0.12 ; \mathrm{Chi}^{2}=19.74, \mathrm{df}=6(\mathrm{P}=0.003) ; \mathrm{l}^{2}=70 \% \\
\text { Test for overall effect: } Z=1.42(\mathrm{P}=0.16)\end{array}$} \\
\hline 1.1.4 Lutein/zeaxant & & & & & & \\
\hline Bosetti 2004 & -0.0943 & 0.1411 & $2.9 \%$ & $0.91[0.69,1.20]$ & $\longrightarrow$ & \\
\hline Gill 2009 & 0.0773 & 0.2035 & $2.2 \%$ & $1.08[0.73,1.61]$ & - & \\
\hline Gill 2009 & 0.708 & 0.4353 & $0.9 \%$ & $2.03[0.86,4.76]$ & & \\
\hline Goodman 2003 & -0.1512 & 0.2923 & $1.5 \%$ & $0.86[0.48,1.52]$ & & - \\
\hline Goodman 2003 & -0.2485 & 0.3021 & $1.4 \%$ & $0.78[0.43,1.41]$ & & \\
\hline Jian 2004 & -3.7987 & 0.7633 & $0.3 \%$ & $0.02[0.01,0.10]$ & & \\
\hline Peters 2007 & -0.0834 & 0.1657 & $2.6 \%$ & $0.92[0.66,1.27]$ & - & - \\
\hline Peters 2007 & -0.0202 & 0.1709 & $2.6 \%$ & $0.98[0.70,1.37]$ & & - \\
\hline Wu 2004 & -0.1863 & 0.2667 & $1.7 \%$ & $0.83[0.49,1.40]$ & & \\
\hline Subtotal (95\% Cl) & & & $16.2 \%$ & $0.86[0.64,1.15]$ & & \\
\hline $\begin{array}{l}\text { Heterogeneity: Tau }{ }^{2} \\
\text { Test for overall effect }\end{array}$ & $\begin{array}{l}0.13 ; \text { Chi }^{2}=28.26 \\
Z=1.01(P=0.31)\end{array}$ & $d f=8(P$ & $=0.000$ & 4); $\left.\right|^{2}=72 \%$ & & \\
\hline 1.1.5 Lycopene & & & & & & \\
\hline Beilby 2010 & -0.2614 & 0.3299 & $1.3 \%$ & $0.77[0.40,1.47]$ & & - \\
\hline Bosetti 2004 & -0.0619 & 0.1372 & $3.0 \%$ & $0.94[0.72,1.23]$ & - & - \\
\hline Gill 2009 & -0.2182 & 0.1958 & $2.3 \%$ & $0.80[0.55,1.18]$ & & \\
\hline Gill 2009 & 0.6206 & 0.5352 & $0.6 \%$ & $1.86[0.65,5.31]$ & & \\
\hline Goodman 2003 & 0.0392 & 0.2713 & $1.7 \%$ & $1.04[0.61,1.77]$ & & \\
\hline Jian 2004 & -1.7148 & 0.42 & $0.9 \%$ & $0.18[0.08,0.41]$ & & \\
\hline Key 1997 & -0.0726 & 0.1901 & $2.4 \%$ & $0.93[0.64,1.35]$ & - & - \\
\hline Kirsh 2006 & -0.1267 & 0.0747 & $3.7 \%$ & $0.88[0.76,1.02]$ & - & \\
\hline Kirsh 2006 & 0.001 & 0.1092 & $3.3 \%$ & $1.00[0.81,1.24]$ & & \\
\hline Kirsh 2006 & -0.2357 & 0.0994 & $3.4 \%$ & $0.79[0.65,0.96]$ & $\rightarrow$ & \\
\hline Nordström 2016 & 0.2007 & 0.3444 & $1.2 \%$ & $1.22[0.62,2.40]$ & & \\
\hline Norrish 2000 & -0.2718 & 0.2192 & $2.1 \%$ & $0.76[0.50,1.17]$ & & \\
\hline Peters 2007 & 0.131 & 0.1665 & $2.6 \%$ & $1.14[0.82,1.58]$ & & \\
\hline Peters 2007 & -0.0101 & 0.2353 & $1.9 \%$ & $0.99[0.62,1.57]$ & & \\
\hline Wu 2004 & -0.4155 & 0.2753 & $1.6 \%$ & $0.66[0.38,1.13]$ & & \\
\hline $\begin{array}{l}\text { Subtotal }(95 \% \mathrm{Cl}) \\
\text { Heterogeneity } \mathrm{Tau}^{2}=\end{array}$ & $0.02 \cdot \mathrm{Chi}^{2}=24.93$ & $d f=14$ & $\begin{array}{l}32.0 \% \\
=0.04\end{array}$ & $\begin{array}{l}0.88[0.78,1.00] \\
I^{2}=44 \%\end{array}$ & & \\
\hline $\begin{array}{l}\text { Heterogeneity: Tau }{ }^{2} \\
\text { Test for overall effect }\end{array}$ & $\begin{array}{l}0.02 ; C h i^{2}=24.93 \\
Z=1.97(P=0.05)\end{array}$ & $d f=14$ & $(P=0.04)$ & $;\left.\right|^{2}=44 \%$ & & \\
\hline Total $(95 \% \mathrm{Cl})$ & & & $100.0 \%$ & $0.88[0.81,0.96]$ & $\bullet$ & \\
\hline $\begin{array}{l}\text { Heterogeneity: } \operatorname{Tau}^{2} \\
\text { Test for overall effect }\end{array}$ & $\begin{array}{l}0.05 ; \mathrm{Chi}^{2}=116.2 \\
Z=2.83(\mathrm{P}=0.005\end{array}$ & $\begin{array}{l}\text { 1. } d f=5 \\
\text { 5) }\end{array}$ & $(P<0.0$ & $0001) ;\left.\right|^{2}=56 \%$ & $\begin{array}{cc}1 & 1 \\
0.05 & 0.2 \\
\text { Favours [experimental] }\end{array}$ & \begin{tabular}{|c|}
1 \\
1 \\
Favours [control]
\end{tabular} \\
\hline
\end{tabular}

Figure 2: Forest plot for the association between Carotenoids and PC risk

\subsubsection{Lutein/Zeaxanthin}

Aiming to investigate the relationship between lutein/zeaxanthin and PC risk, the summary of ORs of six case-control studies with nine datasets was estimated. We observed a statistically non-significant decrease in PC risk with an OR of $0.86(95 \% \mathrm{Cl} \quad 0.64-1.15)$ with high heterogeneity $\left(\mathrm{I}^{2}=72 \%\right)$. A slight decrease and yet statistically not-significant was noticed within North American populations and for studies using serum concentrations of lutein/zeaxanthin to investigate the association ( $\mathrm{OR}=0.96 ; 95 \% \mathrm{Cl} 0.81-1.14)$. 


\subsection{5 $\beta$-cryptoxanthin}

In a pooled analysis of six case-control studies reporting data about the effect of $\beta$-cryptoxanthin on PC risk, an OR of $0.80\left(95 \% \mathrm{Cl} 0.58-1.09, \mathrm{I}^{2}=70 \%\right)$ was found, indicating a statistically non-significant inverse association. A slight decrease and yet statistically not-significant was noticed within North American populations and for studies using serum concentrations of $\beta$-cryptoxanthin to investigate the association $(\mathrm{OR}=0.98 ; 95 \% \mathrm{Cl} 0.80-1.20)$.

\subsection{Publication bias}

The funnel plots put in the Supplementary figures show no sign of asymmetry. Among the five meta-analyses, the risk of bias assessment was available for two analyses since they included a number of datasets $\geq 10$. Both analyses showed no evidence of small study effect. The analysis of lycopene and $P C$ (Begg's test $P=0.9605$, Egger's test $P=0.678$ ) and the analysis of $\beta$-Carotene and PC (Begg's test $P=0.216$, Egger's test $P=0.522$ ).

\section{DISCUSSION}

The current meta-analysis, which included 13 studies, provided evidence that carotenoids could prevent against PC. To sum up, the overall analysis demonstrated a significant decrease in PC incidence. The same pattern was noticed for all types of carotenoids investigated in our study i.e. lycopene $\beta$-carotene, $\alpha$-carotene, $\beta$ cryptoxanthin, and lutein/zeaxanthin.

In vitro and animal, studies have provided evidence that carotenoids may protect against several types of cancer [31-34]. Additionally, the World Cancer Research Fund (WCRF) released a comprehensive report on food and cancer prevention in 2007 highlighting that foods containing carotenoids are probably protective against lung, mouth, pharynx, and larynx cancers [35].

Stahl and Sies [36] stated that carotenoids and/or their metabolites influence the expression of certain genes or may act as regulatory enzymes inhibitors in context with cancer preventive properties of these compounds.

The mechanism underlying the beneficial effect of lycopene on PC risk can be explained by this carotenoid high biological activity through antioxidant properties as preventing DNA damage by scavenging free radicals [8] and modulating gene expression related to PC growth [37]. Furthermore, Yang et al., [38] showed that this nonprovitamin A carotenoid inhibits PC cell proliferation via the PPARy-LXR $\alpha$ - ABCA1 pathway. According to Stahl and Sies [36] and Gerster [39], lycopene was found to hamper the progression of PC via apoptosis induction and angiogenesis suppression.
The current analysis provides support for future studies using carotenoid-based dietary or supplementation interventions and plays a key role in PC survivorship including treatment.

Concerning the risk of $\mathrm{PC}$, our results agree with some previous studies. Wang et al. [40], reported in a metaanalysis of 34 studies, that lycopene $(R R=0.88 ; 95 \% \mathrm{Cl}$ $0.76-1.02)$, but not $\beta$-carotene ( $R R=0.90 ; 95 \% \mathrm{Cl} 0.81-1.01)$, were inversely associated with the risk of PC.

Nonetheless, both Chen et al. (2015) [41], Ilic et al. [42] found a positive association between lycopene consumption $(\mathrm{RR}=0.91 ; 95 \% \mathrm{Cl} 0.81-1.01)$ and $(\mathrm{OR}=-0.34$; $95 \% \mathrm{Cl}-2.01-1.32)$ respectively with the risk of $\mathrm{PC}$.

Controversially to our analysis, Wang et al. [40] stated that $\alpha$-carotene $(\mathrm{RR}=0.87 ; 95 \% \mathrm{Cl} 0.76-0.99)$ was positively associated with the risk of developing PC.

However, similar to dietary carotene intake, Van Hoang et al. [43] observed in a case-control study no relationship between $\beta$-cryptoxanthin intake as well as lutein and zeaxanthin with adjusted ORs of 1.29 (95\% Cl 0.79-2.09) and 0.73 (95\% Cl 0.44-1.20) respectively with $\mathrm{PC}$ risk.

The type of studies (cohort and case-control), populations (Asia, Europe, Australia, and the USA) slightly influenced the odds estimate. However, sensitivity analysis further showed that when omitting the studies conducted by (Jian et al., 2004) [30], the results noticeably changed.

To the best of our knowledge, this is the first meta-analysis undertaken to highlight the relationship between carotenoids and PC risk. However, some limitations should be considered as the limited number of articles included in our analysis. Moreover, our analyses showed heterogeneity evidence. However, subgroup analysis demonstrated sources of heterogeneity that could be attributable to including different study designs in the analyses.

\section{CONCLUSION}

The current meta-analysis has been undertaken to assess available evidence for treatment and prevention of PC, to show the contribution of carotenoids, their intake or supplementation, and to perform a comparison between their relationships with $\mathrm{PC}$ risk.

Our results showed that all types of carotenoids investigated in our study may contribute to the prevention of PC. To determine whether they are clinically relevant, more laboratory and clinical trials are needed on the effects of these compounds on the behavior of prostate cancer cells and tissues. Moreover, the lack of evidence and data supports the call for publishing more research in this area to investigate the effectiveness of carotenoids for the prevention and treatment of PC. 


\section{REFERENCES}

1. Bray F, Ferlay J, Soerjomataram I, Siegel RL, Torre LA, Jemal A. Global cancer statistics 2018: GLOBOCAN estimates of incidence and mortality worldwide for 36 cancers in 185 countries. CA Cancer J. Clin. 2018;68(6). doi:10.3322/caac.21492

2. Ferlay J, Colombet M, Soerjomataram I, Mathers C, Parkin DM, Piñeros M, Znaor A, Bray F. Estimating the global cancer incidence and mortality in 2018: GLOBOCAN sources and methods. Int. J. Cancer. 2018;0(0). doi:10.1002/ijc.31937

3. O'Neill ME, Carroll Y, Corridan B, Olmedilla B, Granado F, Blanco I, Van den Berg H, Hininger I, Rousell AM, Chopra M, Southon S, Thurnham DI. A European carotenoid database to assess carotenoid intakes and its use in a five-country comparative study. $\mathrm{Br}$. J. Nutr. 2001;85(4):499-507. doi:10.1079/BJN2000284

4. Gaziano JM, Manson JE, Branch LG, Colditz GA, Willett WC, Buring JE. A prospective study of consumption of carotenoids in fruits and vegetables and decreased cardiovascular mortality in the elderly. Annals of Epidemiology. 1995;5(4):255-60. doi:10.1016/10472797(94)00090-G

5. Block G, Patterson B, Subar A. Fruit, vegetables, and cancer prevention: A review of the epidemiological evidence. Nutr. Cancer. 1992;18(1):1-29. doi:10.1080/01635589209514201

6. Ziegler RG. A Review of Epidemiologic Evidence that Carotenoids Reduce the Risk of Cancer. J. Nutr. 1989;119(1):116-22. doi:10.1093/jn/119.1.116

7. Hennekens $\mathrm{CH}$. Micronutrients and Cancer Prevention. N. Engl. J. Med. 1986;315(20):1288-89. doi:10.1056/NEJM198611133152009

8. Krinsky NI. The Antioxidant and Biological Properties of the Carotenoids. Ann. N. Y. Acad. Sci. 1998;854(1):443-7. doi:10.1111/j.1749-6632.1998.tb09923.x

9. Bolhassani A. Cancer Chemoprevention by Natural Carotenoids as an Efficient Strategy. Anticancer Agents Med. Chem. 2015;15(8):1026-31. doi:10.2174/1871520615666150302125707

10. Giovannucci E. A Review of Epidemiologic Studies of Tomatoes, Lycopene, and Prostate Cancer. Exp. Biol. Med. $\quad$ (Maywood). 2002;227(10):852-59. doi:10.1177/153537020222701003

11. Tan $\mathrm{H}-\mathrm{L}$, Thomas-Ahner JM, Grainger EM, Wan $\mathrm{L}$, Francis DM, Schwartz SJ, Erdman JW Jr, Clinton SK. Tomato-based food products for prostate cancer prevention: what have we learned? Cancer Metastasis Rev. 2010;29(3):553-68. doi:10.1007/s10555-010-9246-z

12. Peters $U$, Leitzmann MF, Chatterjee $N$, Wang $Y$, Albanes D, Gelmann EP, Friesen MD, Riboli E, Hayes RB. Serum Lycopene, Other Carotenoids, and Prostate
Cancer Risk: A Nested Case-Control Study in the Prostate, Lung, Colorectal, and Ovarian Cancer Screening Trial. Cancer Epidemiol Biomarkers Prev. 2007;16(5):962-68. doi: 10.1158/1055-9965.EPI-06-0861

13. Wu K, Erdman JW, Schwartz SJ, Platz EA, Leitzmann M, Clinton SK, DeGroff V, Willett WC, Giovannucci E. Plasma and Dietary Carotenoids, and the Risk of Prostate Cancer. Cancer Epidemiol Biomarkers Prev. 2004;13(2):260-69. doi:10.1158/1055-9965.EPI-03-0012

14. Moher D, Liberati A, Tetzlaff J, Altman DG, PRISMA Group. Preferred Reporting Items for Systematic Reviews and Meta-Analyses: The PRISMA Statement. PLOS Med. 2009;6(7): e1000097. doi: 10.1371/journal.pmed.1000097

15. Review Manager (RevMan). Version 5.3. Copenhagen: The Nordic Cochrane Centre, The Cochrane Collaboration; 2014.

16. Higgins J, Green S, eds. Cochrane Handbook for Systematic Reviews of Interventions Version 5.1.0 [Updated March 2011]. The Cochrane Collaboration; 2011. www.cochrane-handbook.org.

17. Begg CB, Mazumdar M. Operating Characteristics of a Rank Correlation Test for Publication Bias. Biometrics. 1994;50(4):1088-1101. doi:10.2307/2533446

18. Egger M, Davey Smith G, Schneider M, Minder C. Bias in meta-analysis detected by a simple, graphical test. Br. Med. J. 1997;315(7109):629-34 doi:10.1136/bmj.315.7109.629

19. R Core Team. R: A Language and Environment for Statistical Computing. Vienna, Austria: R Foundation for Statistical Computing; 2018. https://www.rproject.org/.

20. Gill JK, Franke AA, Morris J, Cooney RV, Wilkens LR, Le Marchand L, Goodman MT, Henderson BE, Kolonel LN. Association of selenium, tocopherols, carotenoids, retinol, and 15-isoprostane $\mathrm{F}(2 \mathrm{t})$ in serum or urine with prostate cancer risk: the multiethnic cohort. Cancer Causes Control. 2009;20(7):1161-71. doi:10.1007/s10552009-9304-4

21. Goodman GE, Schaffer S, Omenn GS, Chen C, King I. The Association between Lung and Prostate Cancer Risk, and Serum Micronutrients. Cancer Epidemiol Biomarkers Prev. 2003;12(6):518-26. PMID: 12814997

22. Cook NR, Stampfer MJ, Ma J, Manson JE, Sacks FM, Buring $J E_{1}$ Hennekens $\mathrm{CH}$. $\beta$-carotene supplementation for patients with low baseline levels and decreased risks of total and prostate carcinoma. Cancer. 2000;86(9):1783-92. doi:10.1002/(SICI)10970142(19991101)86:9 <1783:.AID-CNCR21>3.0.CO;2-N

23. Kirsh VA, Mayne ST, Peters U, Chatterjee N, Leitzmann MF, Dixon LB, Urban DA, Crawford ED, Hayes RB. A Prospective Study of Lycopene and Tomato Product Intake and Risk of Prostate Cancer. Cancer Epidemiol 
Biomarkers Prev. 2006;15(1):92-8. doi:10.1158/10559965.EPI-05-0563

24. Nordström T, Van Blarigan EL, Ngo V, Roy R, Weinberg V, Song X, Simko J, Carroll PR, Chan JM, Paris PL. Associations Between Circulating Carotenoids, Genomic Instability and the Risk of High-Grade Prostate Cancer. Prostate. 2016;76(4):339-48. doi:10.1002/pros.23125

25. Beilby J, Ambrosini GL, Rossi E, de Klerk NH, Musk AW. Serum levels of folate, lycopene, $\beta$-carotene, retinol and vitamin $\mathrm{E}$ and prostate cancer risk. Eur J Clin Nutr. 2010; 64(10)1235-8. doi:10.1038/ejen.2010.124

26. Ambrosini $\mathrm{GL}$, de Klerk NH, Fritschi $\mathrm{L}$, Mackerras $\mathrm{D}$, Musk B. Fruit, vegetable, vitamin A intakes, and prostate cancer risk. Prostate Cancer Prostatic Dis. 2008; 11(1):61-6. doi:10.1038/sj.pcan.4500979

27. Norrish AE, Jackson RT, Sharpe SJ, Skeaff CM. Prostate Cancer and Dietary Carotenoids. Am. J. Epidemiol. 2000;151(2):119-23. doi:10.1093/oxfordjournals.aje.a010176

28. Bosetti C, Talamini R, Montella M, Negri E, Conti E, Franceschi S, La Vecchia C. Retinol, carotenoids and the risk of prostate cancer: A case-control study from Italy. Int. J. Cancer. 2004;112(4):689-92. doi:10.1002/ijc.20486

29. Key TJ, Silcocks PB, Davey GK, Appleby PN, Bishop DT. A case-control study of diet and prostate cancer. Br. J. Cancer. 1997;76(5):678-87. doi:10.1038/bjc.1997.445

30. Jian L, Du C-J, Lee AH, Binns CW. Do dietary lycopene and other carotenoids protect against prostate cancer? Int. J. Cancer. 2004;113(6):1010-14. doi:10.1002/ijc.20667

31. Mayne ST. Beta-carotene, carotenoids, and disease prevention in humans. FASEB J. 1996;10(7):690-701. doi:10.1096/fasebj.10.7.8635686

32. Tan H-L, Thomas-Ahner JM, Moran NE, Cooperstone $J$, Erdman JW Jr, Young GS, Clinton SK. $\beta$-Carotene 9',10' Oxygenase Modulates the Anticancer Activity of Dietary Tomato or Lycopene on Prostate Carcinogenesis in the TRAMP Model. Cancer Prev. Res. (Phila). 2017;10(2):161-9. doi:10.1158/1940-6207.CAPR15-0402

33. Assar EA, Vidalle MC, Chopra M, Hafizi S. Lycopene acts through inhibition of I $\mathrm{kB}$ kinase to suppress NF$\mathrm{KB}$ signaling in human prostate and breast cancer cells. Tumor Biol. 2016;37(7):9375-85. doi:10.1007/s13277$\underline{016-4798-3}$
34. Holzapfel NP, Holzapfel BM, Theodoropoulos C, Kaemmerer E, Rausch T, Feldthusen J, Champ S, Clements JA, Hutmacher DW, Loessner D. Lycopene's Effects on Cancer Cell Functions within Monolayer and Spheroid Cultures. Nutr. Cancer. 2016;68(2):350-63. doi:10.1080/01635581.2016.1150498

35. Wiseman M. The Second World Cancer Research Fund/American Institute for Cancer Research Expert Report. Food, Nutrition, Physical Activity, and the Prevention of Cancer: A Global Perspective: Nutrition Society and BAPEN Medical Symposium on 'Nutrition support in cancer therapy.' Proc. Nutr. Soc. 2008;67(3):253-6. doi:10.1017/S002966510800712X

36. Stahl W, Sies H. Bioactivity and protective effects of natural carotenoids. Biochim. Biophys. Acta. 2005;1740(2):101-7. doi:10.1016/j.bbadis.2004.12.006

37. Rafi MM, Kanakasabai S, Reyes MD, Bright JJ. Lycopene modulates growth and survival associated genes in prostate cancer. J. Nutr. Biochem. 2013;24(10):1724-34. doi:10.1016/j.jnutbio.2013.03.001

38. Yang C-M, Lu Y-L, Chen H-Y, Hu M-L. Lycopene and the LXR $\alpha$ agonist T0901317 synergistically inhibit the proliferation of androgen-independent prostate cancer cells via the PPAR $\mathrm{-}$-LXR $\alpha$-ABCA1 pathway. J. Nutr. Biochem. 2012;23(9):1155-62. doi:10.1016/j.jnutbio.2011.06.009

39. Gerster H. Anticarcinogenic Effect of Common Carotenoids. Int. J. Vitam. Nutr. Res. 1993; 63(2):39-121. PMID: 8407171

40. Wang Y, Cui R, Xiao Y, Fang J, Xu Q. Effect of Carotene and Lycopene on the Risk of Prostate Cancer: A Systematic Review and Dose-Response Meta-Analysis of Observational Studies. PLoS One. $2015 ; 10(9)$ : e0137427. doi:10.1371/journal.pone.0137427

41. Chen P, Zhang W, Wang X, Zhao K, Negi DS, Zhuo L, Qi M, Wang X, Zhang X. Lycopene and Risk of Prostate Cancer: A Systematic Review and Meta-Analysis. Medicine (Baltimore). 2015;94(33): e1260. doi:10.1097/MD.0000000000001260

42. Ilic D, Forbes KM, Hassed C. Lycopene for the prevention of prostate cancer. Cochrane Database Syst Rev. 2011;(11) :CD008007 doi:10.1002/14651858.CD008007.pub2

43. Van Hoang D, Pham NM, Lee AH, Tran DN, Binns CW. Dietary Carotenoid Intakes and Prostate Cancer Risk: A Case-Control Study from Vietnam. Nutrients. 2018;10(1) pii: E70. doi:10.3390/nu10010070

Cite this article as: Benkhadda H, Bouchentouf M and El Herrag S.E. Association between carotenoids and prostate cancer risk: A Meta-Analysis. Nor. Afr. J. Food Nutr. Res. January - June (2019); 03 (05): 131-39. https://doi.org/10.5281/zenodo.2567568

Nor. Afr. J. Food Nutr. Res. | January - June 2019 | Volume 03 || Issue 05 